\title{
Hydrodynamic stability and turbulent transition with the Vreman LES SGS and a modified lattice Boltzmann equation
}

\author{
J. R. Murdock (D) and S. L. Yang (D)
}

Department of Mechanical Engineering-Engineering Mechanics, Michigan Technological University, Houghton, MI, USA

\begin{abstract}
For the evaluation of a broad range of $R e$ in incompressible flows, particularly unsteady and transition regimes, the Vreman subgrid scale model is studied within the framework of a modified lattice Boltzmann equation. A unique multiple relaxation time form which recovers the fully incompressible unsteady Navier-Stokes equations is derived for the D3Q19 lattice. Solutions to the 3D-driven cavity are compared to a number of lattice Boltzmann and Navier-Stokes solutions. Initial simulations demonstrate the vanishing nature of eddy viscosity in the steady laminar regime. Onset of unsteadiness is found between Re 1900 and 1950, matching well with the wealth of literature. At Re 6000, velocity history and complex vortex structures show a transition to turbulence near the domain bottom and front walls while the centre of the domain retains laminar characteristics. By Re 8000 intermittent turbulence has progressed to the domain centre. This range of $R e$ for transition and the flow characteristics are in agreement with the general ranges in literature, with further observations being added here. The Vreman model with an incompressible lattice Boltzmann method is found to be a promising tool for laminarto-turbulent simulation.
\end{abstract}

\section{ARTICLE HISTORY}

Received 6 April 2018

Accepted 30 August 2018

\section{KEYWORDS}

Transition; lattice Boltzmann; incompressible; multiple relaxation time (MRT); large eddy simulation (LES); Vreman subgrid model; hydrodynamic stability

\section{Introduction}

Turbulent transition is one of the more active areas of research in computational fluid dynamics (CFD), and without direct numerical simulation (DNS) this common physical occurrence requires unique treatment. To avoid the computational cost of DNS while retaining large-scale fidelity in the turbulent regime, a family of approaches combining resolution of the largest turbulent structures and modelling of the smallest scales has been developed, termed scale resolving simulation (SRS). Of this family, large eddy simulation (LES) is the highest fidelity as only the smallest, approximately isotropic scales are modelled (Pope, 2000). A modification of this 
high-fidelity approach is employed within the lattice Boltzmann method (LBM) in this study for the transition regime.

LBM approaches CFD from a kinetic theory basis (McNamara \& Zanetti, 1988). While the governing lattice Boltzmann equation (LBE) can take many forms, the most common, and the form of interest here, recovers the Navier-Stokes equations in the hydrodynamic limit. The LBM provides many benefits versus the Navier-Stokes equations for turbulent transition flows, including inherent parallelism, low numerical dispersion/ diffusivity and simple boundary condition treatment (Chen \& Martinez, 1996; Kruger et al., 2017; Marie, Ricot, \& Sagaut, 2009; Tolke, 2009).

Complex flows for CFD methods verification are often obtained with the lid-driven cavity (LDC). Despite simple boundaries, the flow exhibits characteristics common in engineering applications such as strong recirculation zones, varying boundary layer properties, and generating and interaction of a variety of vortex sizes. With the plethora of literature on the LDC, new solvers and methodology approaches can be readily compared to those which are well established. Initial computational studies were performed on a $2 \mathrm{D}$ plane section, representing the centre plane of an approximately infinite width cavity in non-turbulent regimes (Ghia, Ghia, \& Shin, 1982). For verification of 3D solvers and fully turbulent flows (turbulence being a 3D phenomenon), the CFD domain can be laterally extended to a closed cube to allow for flow structures in the additional dimension, a phenomenon present in turbulent flows. Experimental work on this geometry has provided general insights and a spread of data across the regimes (Prasad \& Koseff, 1989).

LES turbulence modelling and the LBM have been tested together in the literature through a number of canonical cases. While verified, much of this research has been performed on fully turbulent flow (Ming, Xiao-Peng, \& Premnath, 2012; Ren, Song, \& Haibao, 2018), performed with a version of LES not suitable for transition (Anupindi, Lai, \& Frankel, 2014; Si \& Shi, 2015), or performed with an LBE which produces a compressibility error (Ming et al., 2012; Ren et al., 2018). To overcome these limitations, several modifications are implemented in the present work to further the study of transition in the LBM framework.

In order to overcome the limitation of the original LES subgrid scale (SGS) model proposed by Smagorinsky in 1963, which fails to adjust accordingly in all locations (Pope, 2000), several variants have been proposed. Two of the more utilised are the Dynamic Smagorinsky and Wall Adapting Local Eddy Viscosity (WALE) SGS which can cover the range of laminar through fully turbulent flow (Germano, Piomelli, Moin, \& Cabot, 1991; Nicoud \& Ducros, 1999). However, these and other dynamic models add notable computational cost and implementation complexity in order to compute an appropriate local eddy viscosity, and can even obtain unphysical 
numerics (Cabot, 1994; Ming et al., 2012). More recently, Vreman has proposed an SGS model which locally varies the eddy viscosity at a cost more similar to the original Smagorinsky SGS model (Vreman, 2004).

Since the LBE actually recovers the compressible Navier-Stokes equations in the low Mach limit, there is an error which scales with Mach number when simulating incompressible flows. Recently, Murdock and Yang have developed an explicit derivation methodology to recover the unsteady incompressible Navier-Stokes equations (Murdock \& Yang, 2016). For high $R e$ flows, the multiple relaxation time (MRT) form provides additional numerical stability and viscosity-independent results (d'Humieres, 1992). For DNS in 2D the unsteady incompressible LBE (iLBE) has been developed into an MRT form (Murdock, Ickes, \& Yang, 2017), and extension to $3 \mathrm{D}$ is desirable for generality and accurate simulation of the role of an additional dimension for turbulent structures.

The objectives of this work are to establish a D3Q19 MRT-iLBE based on the 2D MRT-iLBE, and pair it with the Vreman SGS model to verify the combined solver for verification and further insight into the laminar-totransition process. To accomplish this, the study begins by applying the solver to steady laminar flow in the LDC. Re is increased to find the onset of instability (first Hopf bifurcation), the first critical step in the transition process. Finally, $R e$ is increased until the appearance of chaotic variable histories, indicating turbulence, in order to discover the range of transition and to add to the current sparse literature.

\section{Methodology}

\subsection{The incompressible lattice Boltzmann equation}

The Boltzmann equation describes the statistical properties of materials at the mesoscopic level, a step between microscopic molecular dynamics and macroscopic continuum flow (Harris, 2011). For computational physics simulation, the equation is discretised by first-order finite differencing, and the complex collision operator is linearised (Bhatnagar, Gross, \& Krook, 1954). Focus here is placed on the incompressible form, while a thorough description, derivation and history of the LBM is readily available within (Guo \& Shu, 2013; Kruger et al., 2017).

\subsubsection{Single relaxation time}

Both the standard and incompressible LBE proceed from the same form

$$
f_{i}(\boldsymbol{x}+\delta \boldsymbol{x}, t+\delta t)=f_{i}(\boldsymbol{x}, t)-\frac{1}{\tau}\left(f_{i}-f_{i}^{e q}\right),
$$


where $f_{i}$ is the probability distribution function of particle velocities discretised to $i$ lattice vectors. The single value parameter $\tau$ is related to macroscopic viscosity by

$$
v=\frac{1}{3}\left(\tau-\frac{1}{2}\right) \delta t .
$$

It is the equilibrium distribution term, $f_{i}^{e q}$, which can determine the macroscopic behaviour the LBE will exhibit. The term is based on the Maxwellian distribution function of particle velocities, expanded and truncated at the appropriate terms for hydrodynamics. In the work of Murdock and Yang this term is modified to meet certain physical parameters to not just limit density variation, but remove it to obtain the fully incompressible NavierStokes equations (Murdock \& Yang, 2016), i.e. to satisfy the incompressible mass and momentum conservation laws. The conservation principles which constrain portions of the final distribution form are the moments of discrete particle velocity, $\boldsymbol{c}_{i}$, mass, momentum and momentum flux tensor,

$$
\sum_{i} f_{i}=1, \quad \sum_{i} c_{i} f_{i}=\boldsymbol{u}, \quad \sum_{i} c_{i} c_{i} f_{i}=P \boldsymbol{I}+\boldsymbol{u} \boldsymbol{u}
$$

respectively, where bold variables are vector quantities, and $\boldsymbol{I}$ is the identity matrix. The additional required constraints are resolved with an ansatz approach. Applying this methodology to the 3D 19 velocity lattice results in the D3Q19 form

$$
\begin{gathered}
f_{i}^{e q}=\left\{\begin{array}{cc}
1-\frac{2}{9} \frac{1}{c^{2}} P+S_{i}, & \text { for } \quad i=0 \\
\frac{1}{54} \frac{1}{c^{2}} P+S_{i}, & \text { for } \quad i=1-6 \\
\frac{1}{108} \frac{1}{c^{2}} P+S_{i}, & \text { for } \quad i=7-18 .
\end{array}\right. \\
S_{i}=\omega_{i}\left[3 \frac{\left(\boldsymbol{c}_{i} \cdot \boldsymbol{u}\right)}{c}+\frac{9}{2} \frac{\left(\boldsymbol{c}_{i} \cdot \boldsymbol{u}\right)^{2}}{c^{2}}-\frac{3}{2} \frac{(\boldsymbol{u} \cdot \boldsymbol{u})}{c^{2}}\right],
\end{gathered}
$$

where $c$ is the lattice speed, taken to be 1 for square lattices, and $\omega_{i}$ are the lattice vector weights $\omega_{0}=\frac{1}{3}, \omega_{1-6}=\frac{1}{18}$ and $\omega_{7-18}=\frac{1}{36}$. Pressure is then a function of velocity squared and not density variation,

$$
P=-\frac{9 c^{2}}{5}\left[\frac{1}{2 c^{2}}(\boldsymbol{u} \cdot \boldsymbol{u})+f_{0}^{e q}-1\right]
$$

Through the Chapman-Enskog multiscale expansion, Murdock and Yang recover the following conservation equations: 


$$
\begin{array}{ll}
\nabla \cdot \boldsymbol{u} & =0 \\
\frac{\partial \boldsymbol{u}}{\partial t}+\nabla \cdot(\boldsymbol{u} \boldsymbol{u}) & =-\nabla P+v \nabla^{2} \boldsymbol{u}
\end{array}
$$

\subsubsection{Multiple relaxation time}

Use of a single relaxation time is the simplest approximation to the effects of molecular collisions. However, the physical reality is that different quantities, or modes, relax towards an equilibrium state at different rates (d'Humieres, 1992). Several are the familiar macroscopic moments such as $\rho$, or are related to recognisable properties such as diagonal terms of the strain rate tensor. By performing collisions in the moment space each mode can have a favourable relaxation time.

Previously, the unique D2Q9 MRT-iLBE form was derived for DNS study (Murdock et al., 2017). In this work the same approach will be taken to develop a distinct incompressible D3Q19 MRT form for the lattice structure of Figure 1. The derivation proceeds from the SRT form, where Equation (4) provides the necessary equilibrium distribution for incompressible physics. Derivation details of the MRT form utilised in this work can be found in Appendix A, including a script for computing key arrays and matrices. The critical $\boldsymbol{m}^{e q}$ values which create the desired macroscopic behaviour are presented in this section as well,

$$
\begin{array}{ccc}
m_{0}^{e q}=1 & m_{7}^{e q}=u_{z} & m_{14}^{e q}=u_{y} u_{z} \\
m_{1}^{e q}=-30+\frac{19}{3} P+19 u^{2} & m_{8}^{e q}=-\frac{2}{3} u_{z} & m_{15}^{e q}=u_{x} u_{z} \\
m_{2}^{e q}=12-3 P-\frac{11}{2} u^{2} & m_{9}^{e q}=2 u_{x}^{2}-u_{y}^{2}-u_{z}^{2} & m_{16}^{e q}=0 \\
m_{3}^{e q}=u_{x} & m_{10}^{e q}=\frac{1}{2}\left(u_{y}^{2}+u_{z}^{2}\right)-u_{x}^{2} & m_{17}^{e q}=0 \\
m_{4}^{e q}=-\frac{2}{3} u_{x} & m_{11}^{e q}=u_{y}^{2}-u_{z}^{2} & m_{18}^{e q}=0 \\
m_{5}^{e q}=u_{y} & m_{12}^{e q}=\frac{1}{2}\left(u_{z}^{2}-u_{y}^{2}\right) & \\
m_{6}^{e q}=-\frac{2}{3} u_{y} & m_{13}^{e q}=u_{x} u_{y} &
\end{array}
$$

It is noted that the values of $m^{e q}$ derived here differ from those presented in previous incompressible MRT derivations (Du \& Shi, 2010). An important deviation is that the current work is based on the unique equilibrium distribution function of Equation (4), a methodology also utilised for transition DNS D2Q9 MRT-iLBE study (Murdock et al., 2017). 


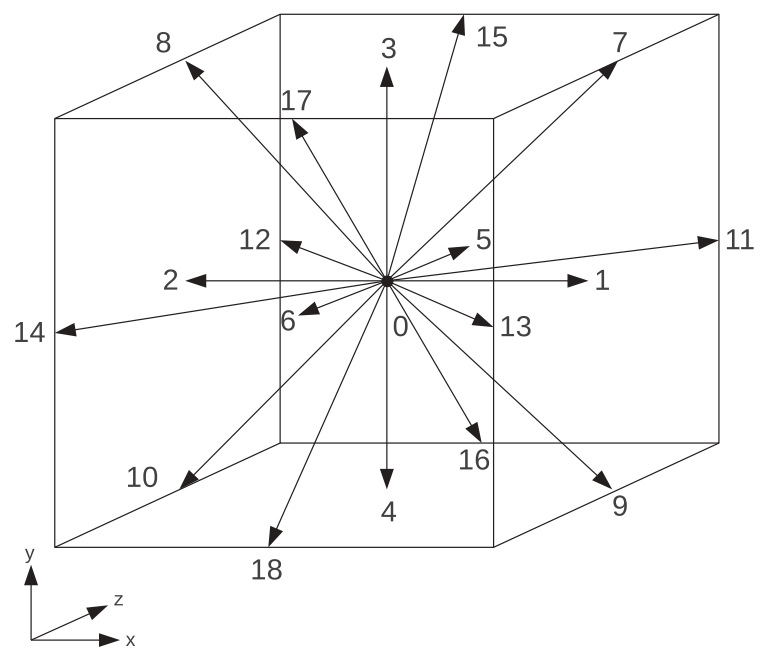

Figure 1. D3Q19 lattice structure used in this study.

\subsection{The Vreman subgrid scale model}

LES offers a unique combination of benefits: reduced overall computational costs due to partial modelling, and DNS-like resolution in the primary energy containing scales (Pope, 2000). However, several researchers have noted the limitations of the original modelling approach to LES. In non-turbulent regimes the eddy viscosity should approach zero, appropriate adjustment should be made in transitional zones, and near walls it should be substantially reduced from the free-stream value. The two primary modifications proposed to correct these shortcomings are the Dynamic Smagorinsky model (Germano et al., 1991), and the WALE model (Nicoud \& Ducros, 1999). Subsequent authors have noted that while the limitations to physicality are reduced by these approaches (although new ones may be introduced (Cabot, 1994)), difficulty in implementation and computational cost arise, and for Vreman, motivated the derivation of a new local computation approach (Vreman, 2004). This relatively new methodology is reviewed here and utilised within the LBM framework.

In the original Smagorinsky approach to LES the modelled viscosity is determined by

$$
v_{e}=\left(C_{S} \Delta\right)^{2}|\overline{\boldsymbol{S}}|,
$$

where $C_{S}$ is a semi-empirically determined value, $|\bar{S}|$ is the magnitude of the filtered strain rate and $\Delta$ is the filter width (Smagorinsky, 1963), often taken to be the grid size and dropped in further notation due to the square lattice of size unity used here. $C_{S}$ is a constant value usually between 0.1 and 0.2. Various authors have arrived at values such as $0.13,0.15$ and 0.17 
based on different analyses; however, the derived value is not valid over all areas of the domain. Ideally, the value would be zero in laminar flows, and substantially lowered near walls. Simplicity is the major benefit of this model, and in fully turbulent flows it is often appropriate.

While the Dynamic Smagorinsky approach alters $C_{S}$ in time and space to ensure an appropriate eddy viscosity, Vreman's approach retains the constant. Eddy viscosity is instead locally modified by employing a different combination of velocity gradients computing not only the strain rate, but also vorticity. Equation (9) displays how the components are combined:

$$
v_{e}=c \sqrt{\frac{B_{\beta}}{\alpha_{i j} \alpha_{i j}}},
$$

where $c$ is a new constant defined as $\approx 2.5 C_{S}^{2}$. For this study $C_{S}$ is 0.1 . Despite the existence of an empirical constant, the square root term provides sufficient local variation, even to a value approaching zero. Additionally, the computation is relatively simple, unlike previous dynamic models, and the components (following the summation convention) are

$$
\alpha_{i j}=\frac{\partial \bar{u}_{j}}{\partial x_{i}}
$$

and

$$
B_{\beta}=\beta_{11} \beta_{22}-\beta_{12}^{2}+\beta_{11} \beta_{33}-\beta_{13}^{2}+\beta_{22} \beta_{33}-\beta_{23}^{2}
$$

where

$$
\beta_{i j}=\alpha_{m i} \alpha_{m j}
$$

The above computations are limited to first-order derivatives, and comparable to the computation cost of $|\bar{S}|$ in the basic Smagorinsky model (Vreman, 2004). In fact, even compared to the WALE model the cost may be as much as $40 \%$ lower (Ming et al., 2012). A further advantage is that $v_{e} \geq 0$ without the need for clipping in order to maintain physicality. Gradients required for the subgrid model are computed with second-order finite differencing.

As in the fully resolved LBE, viscosity is determined by the collision relaxation time. Thus it is straightforward to provide a modelled eddy viscosity component based on the Boussinesq hypothesis:

$$
\tau_{\text {eff }}=3\left(v+v_{e}\right)+\frac{1}{2} .
$$




\section{Lid-driven cavity parameters}

All walls of the six-sided cubic domain of Figure 2 are no-slip. The top wall translates in the positive $x$-direction. Each edge of the domain is given an equivalent length, $L$, used as the characteristic length. The walls are implemented with the second-order accurate halfway bounceback method frequently used in LBM (Chen \& Martinez, 1996). Ladd developed a modification to the bounceback scheme to introduce the momentum source of a Dirichlet boundary condition (Ladd, 1994). This method is utilised, but with a minor modification since density is no longer a variable,

$$
f_{i}(\boldsymbol{x}, t+\delta t)=f_{i^{\prime}}^{\prime}(\boldsymbol{x}, t)-6 \omega_{i^{\prime}} c_{i^{\prime}} \cdot \boldsymbol{u}
$$

where $f^{\prime}$ is the post-collision distribution function, $i^{\prime}$ is the lattice velocity opposite of $i$ and $\boldsymbol{u}$ is the intended velocity at a given boundary condition.

Two boundary conditions have been historically implemented for the translation of the lid: a value $U$ which is constant for all points on the lid, and a regularised value which is dependent on the specific location as

$$
u_{x}\left(x, y_{\text {top }}, z\right)=U\left(1-\left(\frac{2 x}{L}-1\right)^{18}\right)^{2}\left(1-\left(\frac{2 z}{L}-1\right)^{18}\right)^{2}
$$

to deal with the discontinuity at the edges. In the laminar cases the wall translates at a constant, position-independent value $U$ to fully conform to the implementation in relevant literature (Chang, Hong, Lin, \& Lin, 2013; Feldman \& Gelfgat, 2010; Hammami, Ben-Cheikh, Campo, Ben-Beya, \& Lili, 2012; Kuhlmann \& Albensoeder, 2014; Loiseau, Robinet, \& Leriche, 2016; Mynam \& Pathak, 2013; Wong \& Baker, 2002). For turbulence onset

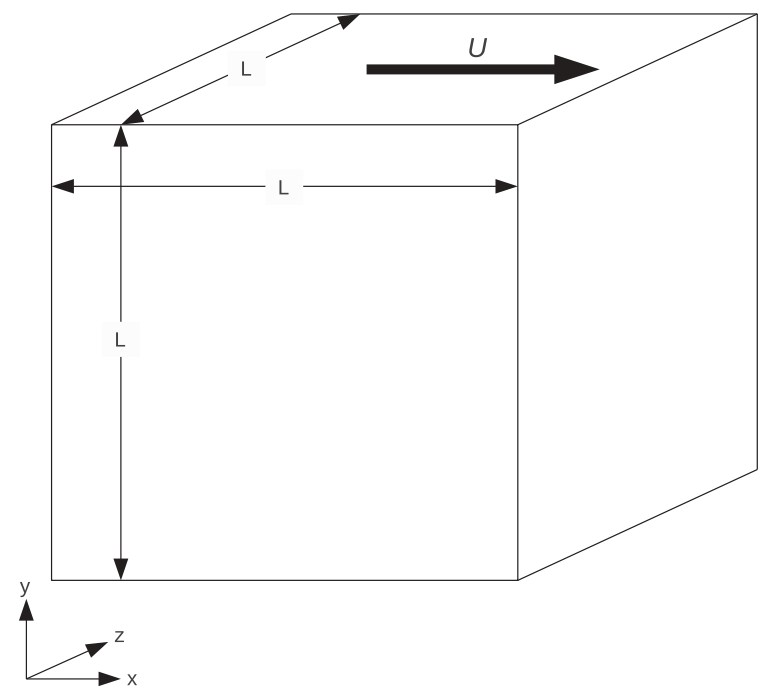

Figure 2. Domain of the 3D LDC. 
the regularised velocity boundary condition, which is common in higher Re simulations (Anupindi et al., 2014; Leriche \& Gavrilakis, 2000; Shen, 1991; Shetty, Fisher, Chunekar, \& Frankel, 2010), is employed.

$R e$ is the independent variable in this simulation, and is defined as

$$
R e=\frac{U L}{v} .
$$

All of the values utilised in the calculation are actually lattice Boltzmann units. Due to similarity the results are equivalent to those obtained through physical units as long as $R e$ is held constant between physical and lattice Boltzmann domains. Additional generality is achieved by presenting results in non-dimensionalised form. For this work $L$ and $U$ are held constant, and $v(\tau)$ is varied to meet the required $R e$. The statements on unit type hold true for the characteristic time as well, defined as

$$
t_{c}=\frac{L}{U},
$$

supplying the value for non-dimensional time calculation.

Simulations are initialised with zero velocity and uniform pressure fields. As a result, a burn in time is required before steady or statistical results are taken. When the flow demonstrates a constant mean value over several hundred characteristic times the results are considered converged. Non-dimensional probe points are monitored for convergence. Data at these points are reported every 10 iterations (10 explicit LBM time units) to avoid aliasing the details of the oscillations. Field results of transient simulations are presented in a time-averaged form.

\section{Results}

\subsection{Steady laminar flow}

To verify the D3Q19 MRT-iLBE proposed in Section 2.1.2, and to validate the low- $R e$ vanishing nature of the Vreman subgrid scale model contribution in the LBM framework simulations are performed at Re 100, 400 and 1000 for comparison to literature (Wong \& Baker, 2002). In all cases the $x$-velocity component is recorded along the vertical centreline of the domain. At this Re it is expected that there is no $z$ velocity component. Results are acquired once the unnormalised RMS residuals of velocity and pressure attain $1.0 \mathrm{e}-9$. A grid-sensitivity study was performed to ensure that the minimum value of $u_{x}$ along the centreline changed by no more $1 \%$ with total grid count increases of at least $50 \%$, resulting in a grid of $50^{3}$ and $L=51$ for data collection.

The pattern of $x$-velocities plotted along the centreline running from the stationary base to the translating lid is compared to 11 points from Navier- 


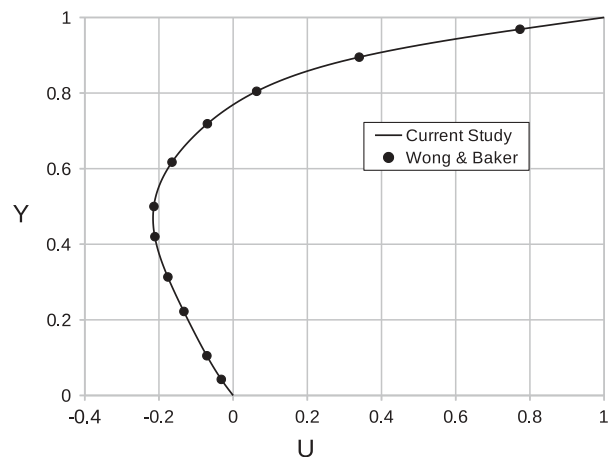

(a) Re 100 centerline $x$-velocity.

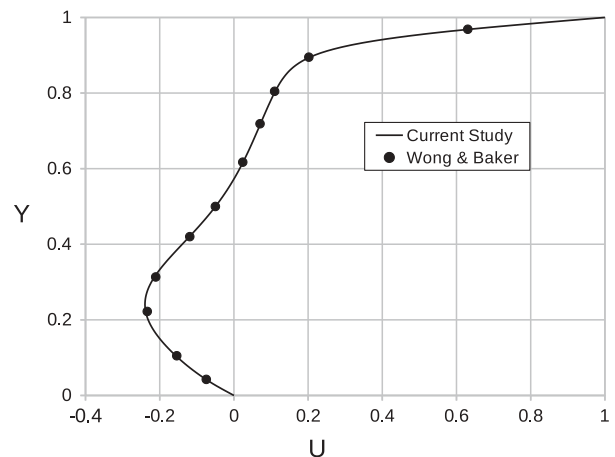

(b) Re 400 centreline $x$-velocity.

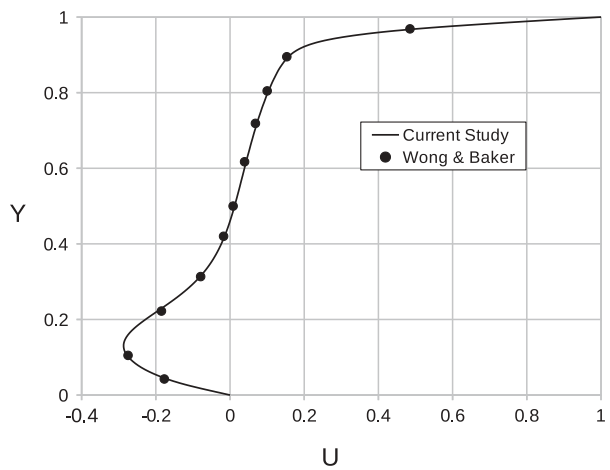

(c) Re 1000 centreline $x$-velocity.

Figure 3. Low Re $x$-velocity along the vertical centreline.

Stokes solutions (Wong \& Baker, 2002) in Figure 3. In all cases the current solver (including the SGS model) matches well with the purely laminar solution, including the velocity variation peaks. $z$-velocity is consistently machine zero at the centre plane as expected for the symmetric solution at these $R e$.

In all cases, the maximum eddy viscosity ratio present in the domain is still dominated by the molecular viscosity $\left(v_{e} / v=1.1 \mathrm{e}-1\right.$ at $\left.\operatorname{Re} 1000\right)$ per the expectation of a laminar simulation. Averaging the value over the whole domain demonstrates that most of the domain experiences virtually no model contribution $\left(v_{e} / v=3.4 \mathrm{e}-3\right.$ at $\left.R e 1000\right)$. The slight contribution of the model is unsubstantial based on the results of Figure 3. Figure 4(c) shows the location of maximum ratio on the centre plane occurs where the main recirculating flow rejoins the flow along the driven wall and where the flow must turn along the wall at $x=1$, indicating a location of high shear.

The location and magnitude of the major vortical structures are an excellent match to the literature evidenced by Figure 4(a). The pressure field of Figure 4 (d) is also qualitatively a good match with the results of other incompressible methods. Kinetic energy in the domain is largely concentrated within a relatively focused zone, visualised in Figure 4(b) where the isosurface contains areas where velocity magnitude is at least $20 \%$ of the maximum. 


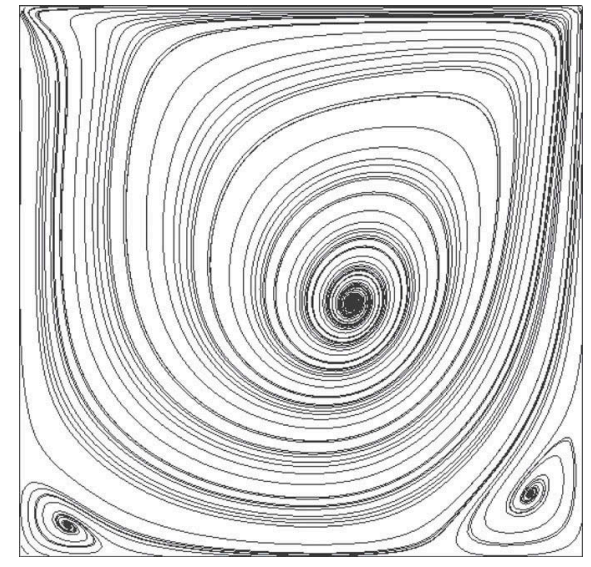

(a) Streamlines.

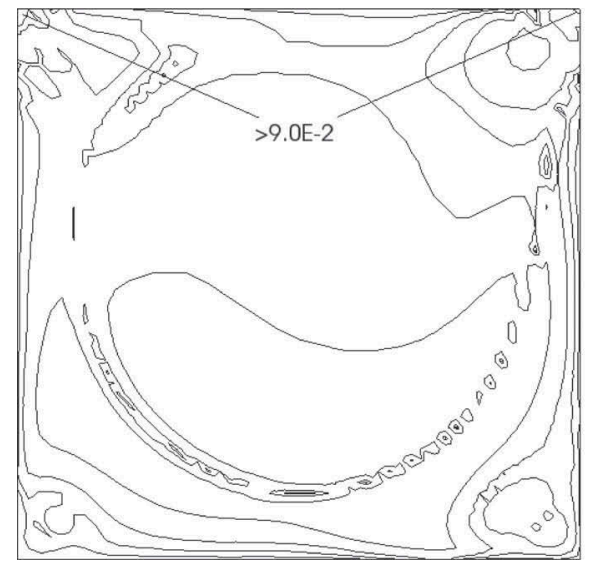

(c) Eddy viscosity ratio.

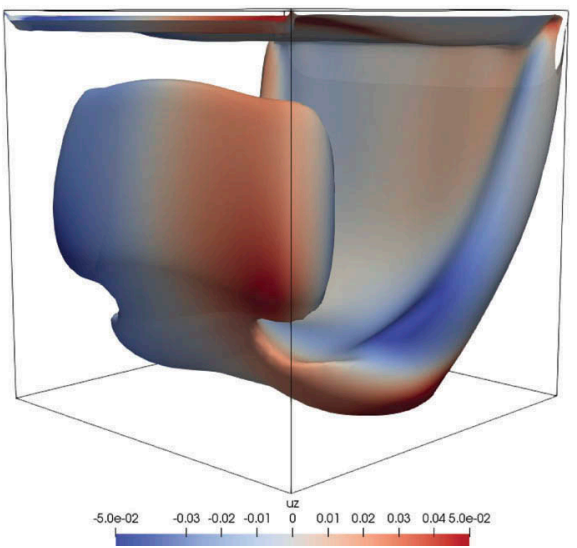

(b) Velocity magnitude (0.2) isosurface, colored by $z$-velocity.

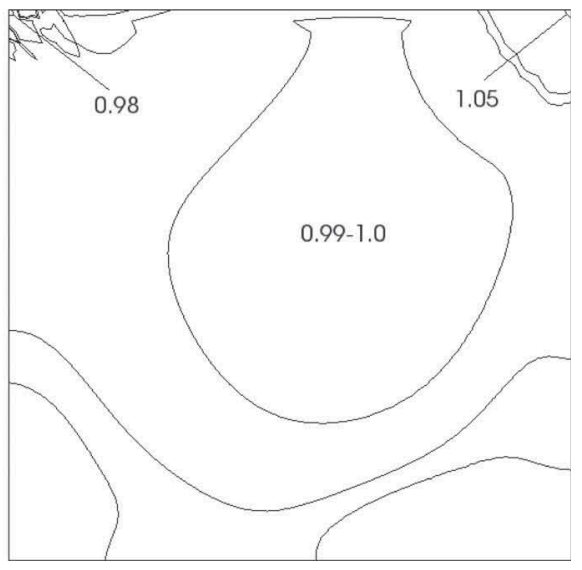

(d) Pressure field.

Figure 4. Re $1000 \mathrm{z}$-centre plane and isosurface results.

\subsection{The first Hopf bifurcation}

With increasing $R e$, instability will arise at a value past those simulated in Section 4.1. This point is well documented in literature for the 3D LDC (Anupindi et al., 2014; Chang et al., 2013; Feldman \& Gelfgat, 2010; Hammami et al., 2012; Kuhlmann \& Albensoeder, 2014; Loiseau et al., 2016; Mynam \& Pathak, 2013), making it a good solver verification tool. The goal here is to provide a narrow set of bounds on $R e$ to estimate $R e_{H o p f}$, where the flow becomes fundamentally transient. Grid size is determined by simulating $R e 1950$ with lattice node counts increasing by at least $50 \%$ until the mean value and amplitude of $u$ changes by no more than $3 \%$, resulting in a grid of $70^{3}$ and $L=71$. A relatively high tolerance is 
employed so the behaviour of the SGS model on hydrodynamic stability can be studied and verified without refining away its calculation.

To measure the onset of unsteadiness a series of $R e$ are chosen based on the previous literature. A given simulation is considered unsteady when the solver cannot achieve the target steady-state RMS velocity residual value of $1.0 \mathrm{e}-6$. Figure 5 displays the time histories of velocity magnitude at probe point $(0.6,0.6,0.5)$, well after initial convergence and transients, between $t_{c} 2000$ and 2100. The first Re plotted, 1900, is on the lower end of the literature consensus of Table 1 and displays a constant converged value. Moving up to $R e 1950$, a regular oscillatory pattern ensues. Several thousand $t_{c} s$ demonstrate that this pattern persists and represents a post-bifurcation flow. The resultant range of values, 1900-1950, represents a good match with the majority of the literature. Despite a comparatively coarse grid, the Vreman SGS, relying on velocity gradient values from the iLBE computation, does not unduly influence the proper solution of the unsteady laminar flow field.

Looking to the time-averaged fields in Figure 6(a)-(d), some similarities to the stable $R e$ results are noted. The main vortex has shifted slightly to the bottom-right corner, but the corner vortices remain in roughly the same position and of the same magnitude. Flow energy is still largely contained within the focused zone of Figure 6(b), although that zone is taking on slightly more topological complexity. Symmetry is also maintained in the unsteady case as $u_{z}$ on the centre plane is machine zero. Eddy viscosity ratio is nearly zero, and the pressure nearly one at the plotted probe point of Figure 5, indicating it is a relative calm zone. Eddy viscosity ratio throughout the domain remains relatively low, with a maximum value of $1.8 \mathrm{e}-1$, and an average of $4.1 \mathrm{e}-3$ at $R e 1950$. The top corners continue to generate both

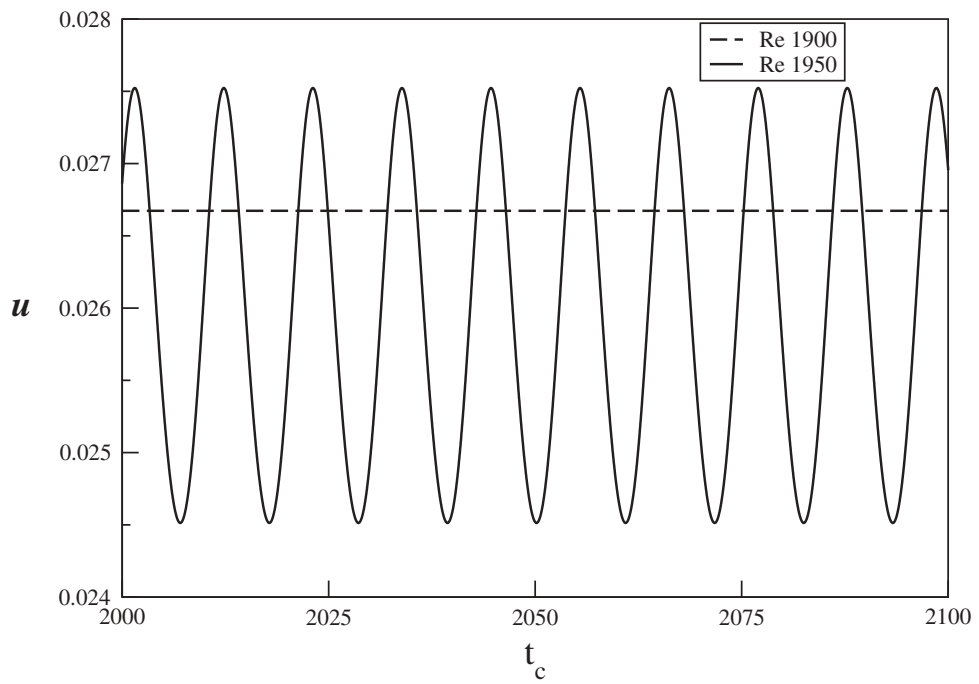

Figure 5. Velocity histories near the Hopf bifurcation at probe point $(0.6,0.6,0.5)$. 
Table 1. Comparison to literary predictions of $R e_{\text {Hopf }}$.

\begin{tabular}{|c|c|c|}
\hline References & Methods summary & $\operatorname{Re}_{\text {Hopf }}$ \\
\hline Feldman and Gelfgat (2010) & $200^{3}$ FVM NS & 1914 \\
\hline Hammami et al. (2012) & $48^{3}$ third-order FVM NS & 1922 \\
\hline Mynam and Pathak (2013) & $301^{3}$ LBM & $1900-2000$ \\
\hline Chang et al. (2013) & $128^{3} \mathrm{LBM}$ & $1750-1950$ \\
\hline Kuhlmann and Albensoeder (2014) & $96^{3}$ Spectral NS & 1919.5 \\
\hline Anupindi et al. (2014) $\dagger$ & $80^{3}$ LES $^{\mathrm{a}}$ LBM & $2100-2250$ \\
\hline Loiseau et al. (2016) & $10^{3}$ Sixth-order spectral NS & $1900-1930$ \\
\hline Present Study & $70^{3}$ LES $^{\mathrm{b}}$ iLBM & $1900-1950$ \\
\hline
\end{tabular}

$\nmid$ Regularised $U,{ }^{\mathrm{a}}$ Smagorinsky SGS, ${ }^{\mathrm{b}}$ Vreman SGS.

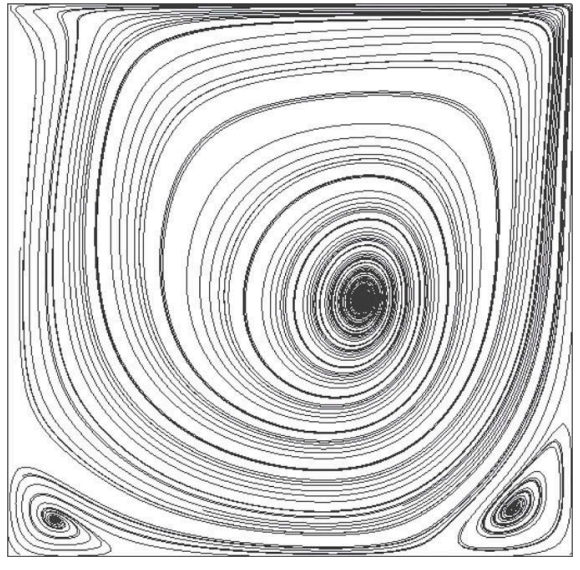

(a)Streamlines.

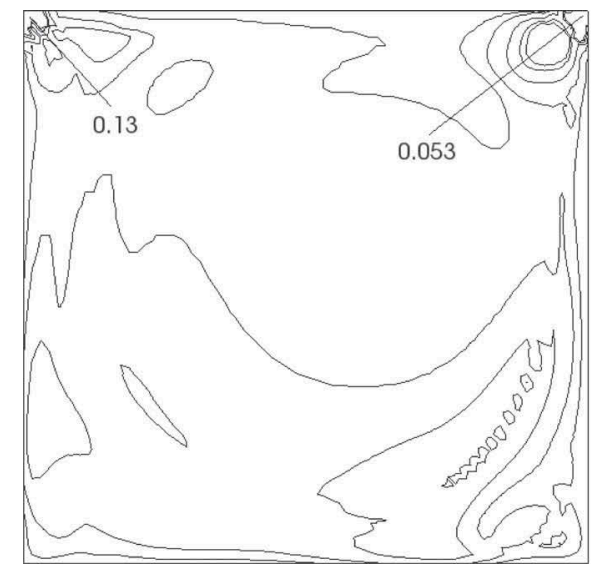

(c)Eddyviscosityratio.

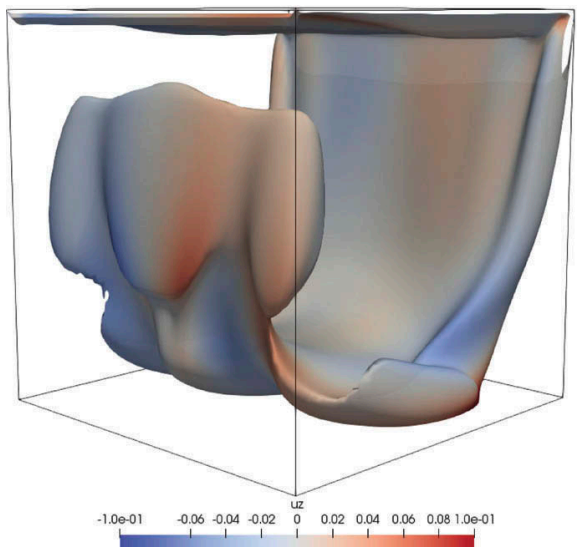

(b) Velocity magnitude (0.2)isosurface, coloredby $z$-velocity.

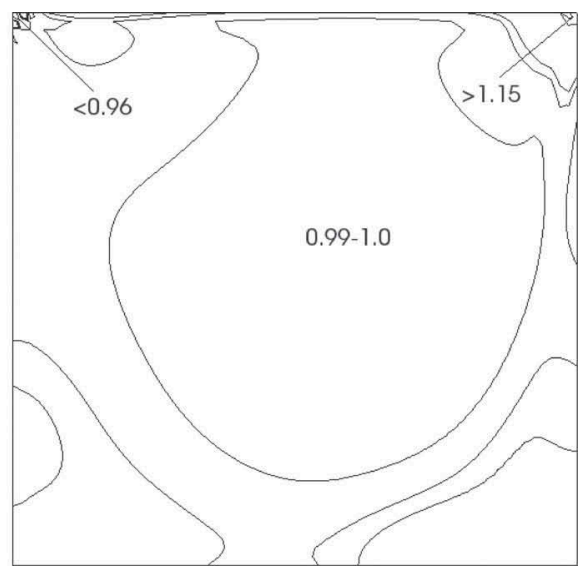

(d)Pressurefield.

Figure 6. Re 1950 time-averaged $z$-centre plane and isosurface results. 
the highest and lowest pressures on the centre plane.

\subsection{Transition to turbulence}

Following the experimental studies of (Prasad \& Koseff, 1989) primitive variable history is tracked to observe the transition to high-frequency fluctuations and trace irregularity telling of turbulence. A probe point $(0.5,0.05,0.5)$ near the bottom of the domain, similar in location to their measurement point, provides data closer to the boundary layer where it is expected that chaos will arise. Additionally, probe point $(0.6,0.6,0.5)$ is tracked as it lies well within the core region, which is expected to see a later transition since different portions of the domain can experience different flow regimes (Shankar \& Deshpande, 2000). To replicate the time ranges visualised in Prasad and Koseff and Shankar and Deshpande for comparative purposes the period starting from 0 to $100 t_{c}$ is inspected for transition, although data are also examined at later times. Prasad and Koseff and Shankar and Deshpande indicate transition occurs roughly between $R e>3200-10,000$ and $R e$ 6000-8000, respectively. Study of transition behaviour with the current methods begins at $R e$ 6000. For post-transition characteristics Bouffanais et al. provide comparative results in the LDC in the unambiguous turbulent regime (Bouffanais, Deville, \& Leriche, 2007).

Lid velocity is governed by the regularised condition. As in previous literature the collision relaxation times not related to viscosity are reduced to 0.7 for stability at the higher $R e$ (Chang et al., 2013). Initial runs for $R e 6000$ were made with $100^{3}, 128^{3}$ and $138^{3}$ points. The $>200 \%$ increase in grid density between $100^{3}$ and $128^{3}$ yields a 5-8\% change in mean velocity and extrema while retaining the same basic trace patterns. Moving to the $138^{3}$ points results in a virtually identical trace pattern with mean and extrema changing by less than $1 \%$. The studies in this section are thus conducted on a $138^{3}$ grid. Characteristic length is then 139. While the SRT collision operator would have been sufficient for the laminar studies, here the flexibility to tune non-viscosity parameters in the MRT form is critical, and with SRT the simulation at $\operatorname{Re} 6000$ diverges almost immediately.

\subsection{1. $\operatorname{Re} 6000$}

Time histories in Figure 7 display two different regimes in the cavity: A laminar core (probe $(0.6,0.6,0.5)$ ) and a turbulent near-bottom zone (probe $(0.5,0.05,0.5))$. The core probe point (Figure $7(\mathrm{a})$ and (c)) takes on the characteristics of a slow and smooth fluctuation after the initial transients. Prasad and Koseff attribute these low-frequency fluctuations to meandering TaylorGoertler-like (TGL) vortices in their Re 3200 case, and given the strong similarities with their Re 3200 study, that is the analysis here. These similarities include amplitude and frequency of the fluctuations, as well as a general 


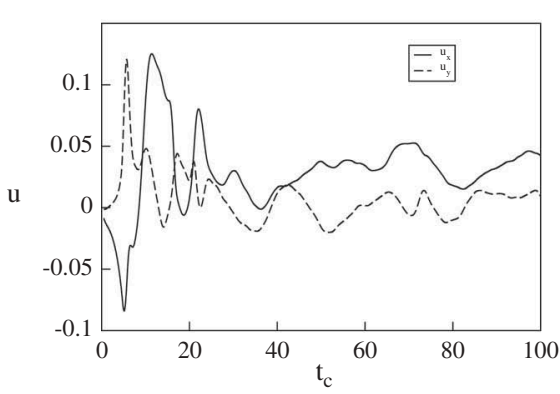

(a) Probe $(0.6,0.6,0.5)$, initial.

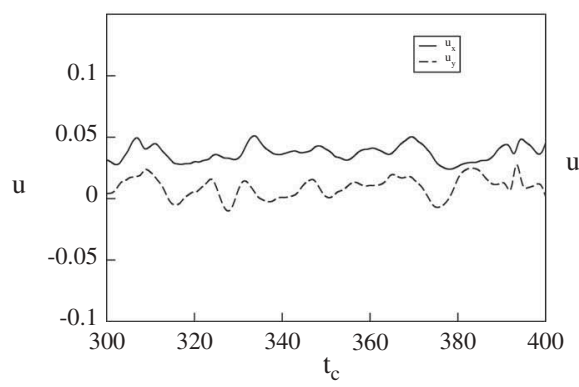

(c) Probe $(0.6,0.6,0.5)$, late.

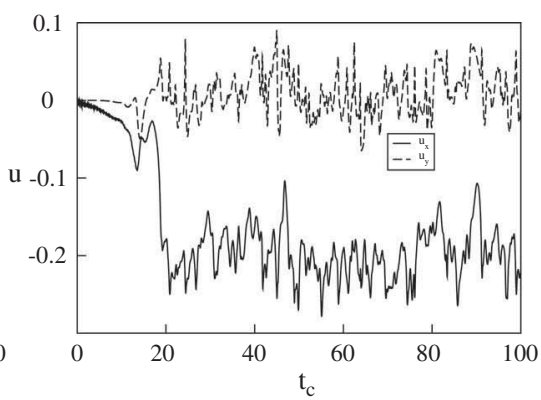

(b) Probe $(0.5,0.05,0.5)$, initial.

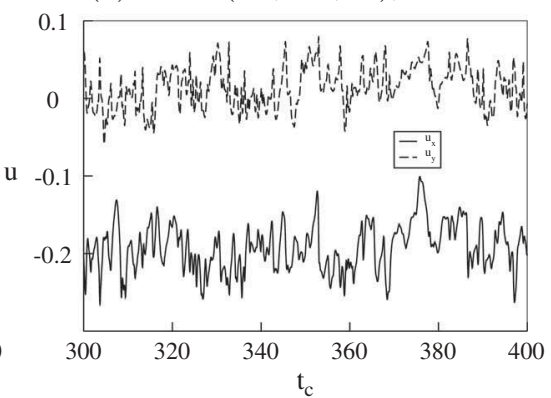

(d) Probe $(0.5,0.05,0.5)$, late.

Figure 7. Re 6000 velocity component time histories.

correlation between component variations. Figure 7(c) goes beyond the characteristic time they present, but shows a clear continuation of the trend. The RMS values, $u_{x^{\prime}}=0.007$ and $u_{y^{\prime}}=0.009$, are close to the Re 3200 values of Prasad and Koseff, demonstrating a calm flow. Symmetry is broken at both points, and at the centre the absolute value of $u_{z}$ fluctuates about one order-ofmagnitude below the $x$ and $y$ components.

Flow at probe $(0.5,0.05,0.5)$ has a very different history in Figure $7(b)$ and $(d)$. The characteristic high-frequency random fluctuations of turbulence are present and persistent, and as a result there is little correlation between velocity components. Velocity histories at this probe bear strong similarities to what Prasad and Koseff present at Re 10,000, and what Bouffanais et al. find at $R e$ 12,000. RMS $u_{x^{\prime}}=0.035$ and $u_{y^{\prime}}=0.027$ are of a similar magnitude to their high-Re turbulent results. Shankar and Deshpande corroborates this transitory behaviour wherein the chaos first develops near the high shear wall layers and is not immediately transferred to the core flow.

At $\operatorname{Re} 6000$ transition to turbulence is underway in the cavity, but not the ubiquitous regime. The Vreman SGS model has contributed to a level that is consistent with the expected results. Maximum eddy viscosity ratio rises to 0.884 , a value representing near equivalence between modelled viscosity and molecular viscosity. Eddy viscosity ratio on the centre plane in Figure 8(c) displays a pattern of higher values along the front and bottom of the cavity where the flow has obtained high energy from the lid and is in shear between 


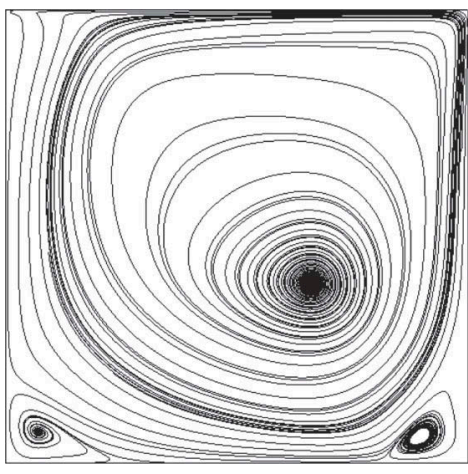

(a)Streamlines.

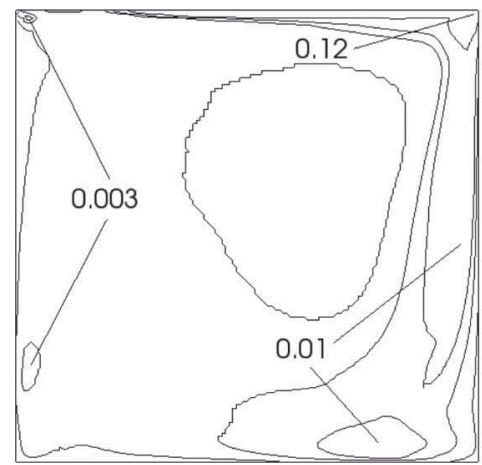

(c)Eddyviscosityratio.

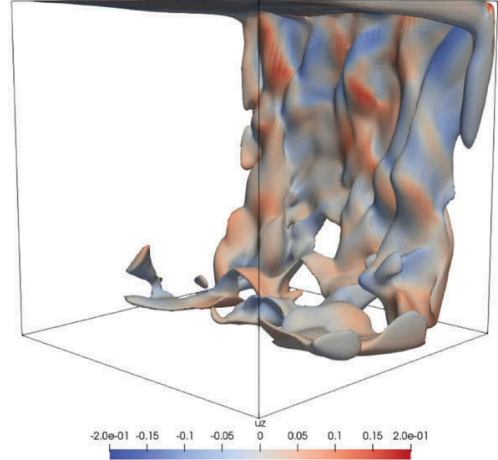

(b) Velocity magnitude (0.2)isosurface, colored by $z$-velocity.

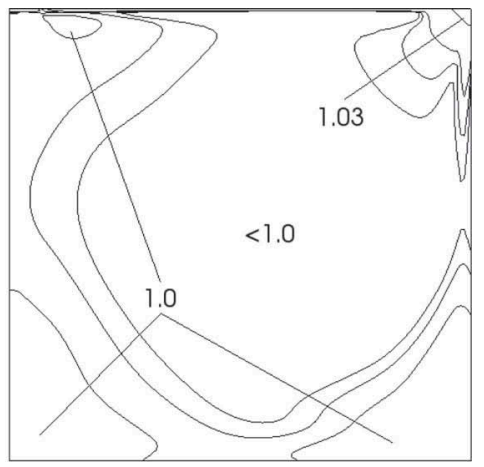

(d)Pressurefield.

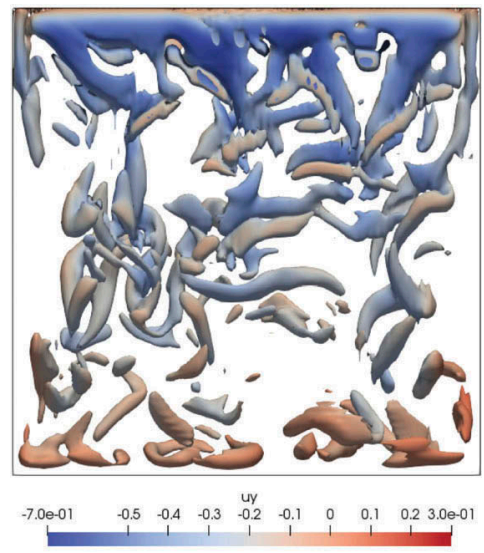

(e)Q-criterion (5)isosurface along front wall, colored by $y$ velocity.

Figure 8. Re 6000 time-averaged $z$-centre plane and instantaneous isosurface results. 
the wall and lower energy core. These areas are also visualised in Figure 8(b) and (e) where the primary kinetic energy and vortical structures are concentrated, although noticeably more dispersed than the low-Re studies.

\subsection{2. $\operatorname{Re} 8000$}

At probe point $(0.5,0.05,0.5)$ little has changed from $\operatorname{Re} 6000$, however the core probe point shows a noticeable shift in Figure 9(a) and (c). As time progresses, higher frequency, seemingly random periods arise intermittently, and at later times constitute a large proportion of the history. Some nonturbulent characteristics are still observable during certain periods, such as relatively smooth changes to velocity, and a general correlation in component changes. Side-by-side with probe $(0.5,0.05,0.5)$, the intermittent bursts are relatively weak, but show strong similarities. Figure 10(a) clearly shows the divergence in behaviour between $R e 6000$ and 8000 via power spectral density (PSD) vs. frequency. At the core probe there is a shift in energy to higher frequencies indicative of turbulence while the lower frequencies are at a similar level. The pattern has changed from a largely linear decline in energy with increasing frequency (on the log-log scale) to a more clearly differentiated intermediate range following the expected $-5 / 3$ power law. This new pattern at $R e 8000$ matches that seen in unambiguously turbulent

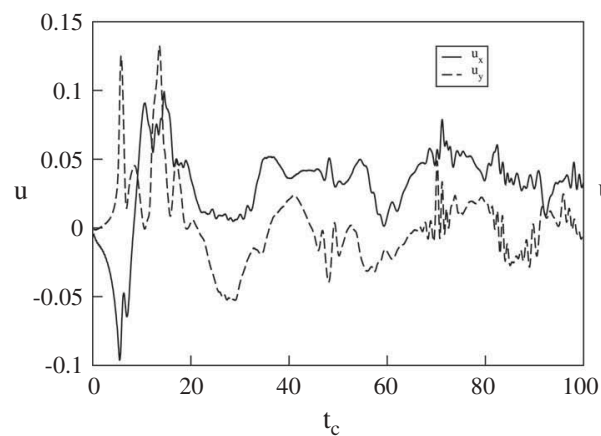

(a) Probe $(0.6,0.6,0.5)$, initial.

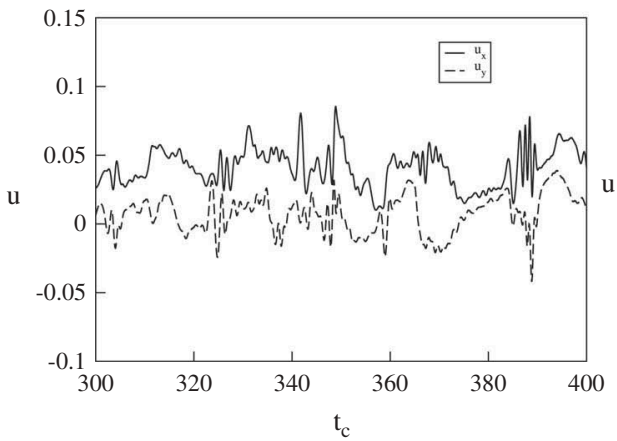

(c) Probe $(0.6,0.6,0.5)$, late.

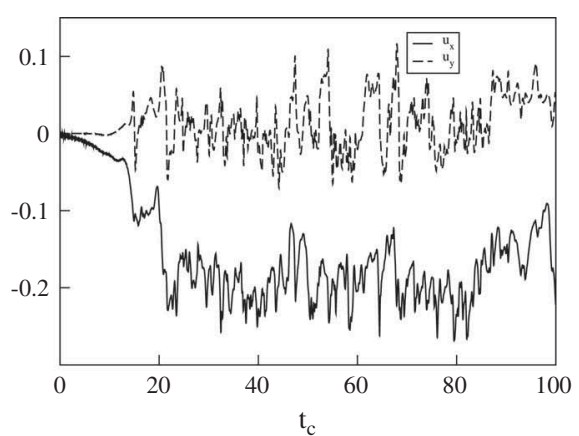

(b) Probe $(0.5,0.05,0.5)$, initial.

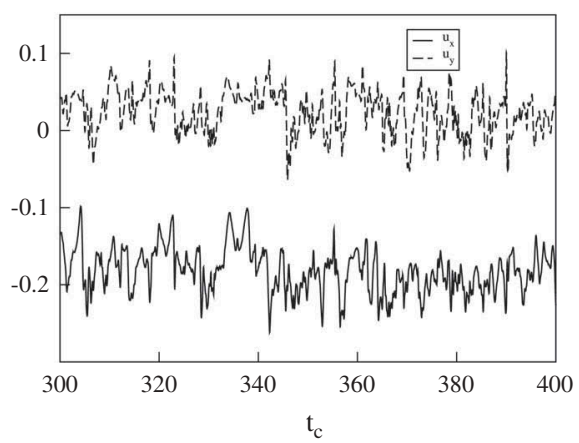

(d) Probe $(0.5,0.05,0.5)$, late.

Figure 9. Re 8000 velocity component time histories. 


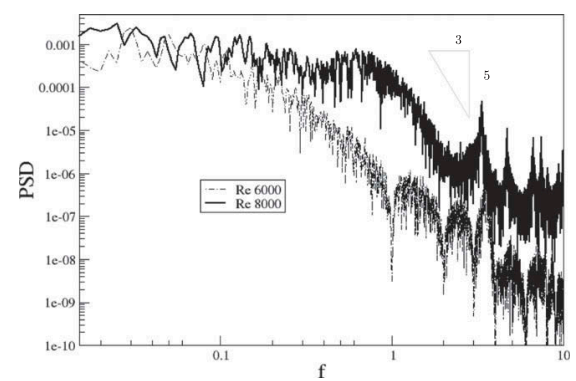

(a) Probe point $(0.6,0.6,0.5)$.

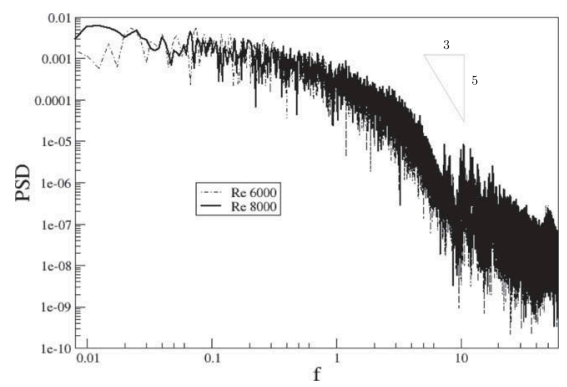

(b) Probe point $(0.5,0.05,0.5)$.

Figure 10. Power spectral densities.

flows (Bouffanais et al., 2007), and compares well against the chaotic flow at probe point $(0.5,0.05,0.5)$ (see Figure $10(\mathrm{~b})$ ). The RMS values at the core probe point, $u_{x^{\prime}}=0.015$ and $u_{y^{\prime}}=0.016$, are doubled from their $R e 6000$ values. While this indicates a move towards turbulence, the more active probe point has values of $u_{x^{\prime}}=0.033$ and $u_{y^{\prime}}=0.029$, which are in keeping with fully turbulent results (Prasad \& Koseff, 1989).

Qualitative changes to the flow are visible in Figure 11(a)-(e). Kinetic energy in the flow, visualised by velocity magnitude isosurface shows a greater dissipation as the area shrinks. Eddy viscosity ratio is at its greatest at the top right corner and along the front wall to the bottom before shear and vorticity drop significantly. In this case the maximum eddy viscosity ratio for the domain rises to 1.38. Plotting the same isosuface value for q-criteria in Figure 11(c) as in the $R e 6000$ case shows a much more active field.

An additional insight and data point is presented in Figure 12, where the time-averaged $x$-velocity values are plotted along the bottom half vertical centreline of the cavity. While the Re values are not identical to those presented in previous literature, comparison to centreline trends in Prasad and Koseff (1989) further verifies the method while adding to the record. The difference in the way velocity was applied experimentally compared to Equation (15) must also be borne in mind. When compared to the Re 5000 and 7500 values from the aforementioned authors the same general patterns emerge: a strong velocity gradient at the bottom of the domain, an inverse relationship between maximum $u$-velocity magnitude and $R e$, and a sharp change in velocity near the bottom. An additional small, but noticeable 'bump' occurs in velocity above the primary sharp change. While the magnitude of the minimum velocity value is lower compared to experiment (due to the way lid velocity is applied), the trend of a decrease in the extrema with increasing $R e$ matches experiment. With the increase in turbulent velocity fluctuations, energy is shifted from the mean momentum. 


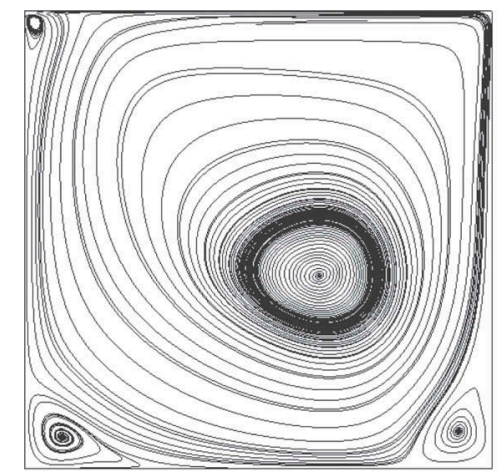

(a) Streamlines.

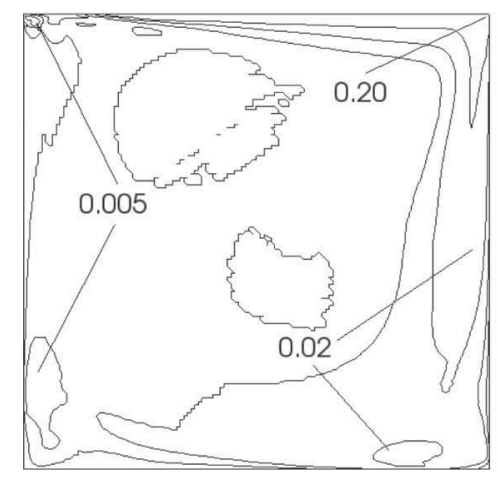

(c) Eddy viscosity ratio.

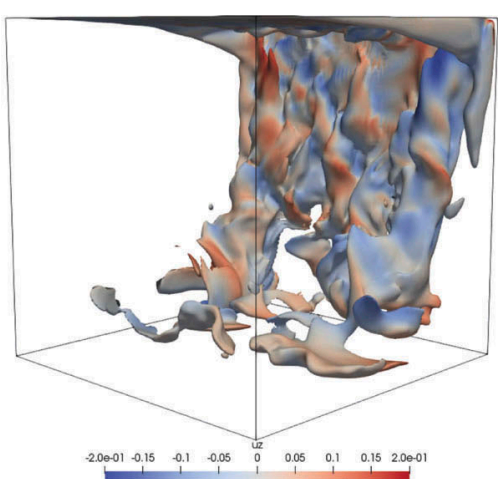

(b) Velocity magnitude (0.2) isosurface, colored by $z$-velocity.

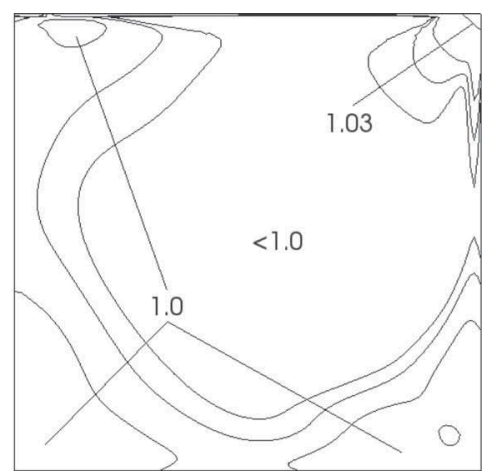

(d) Pressure field.

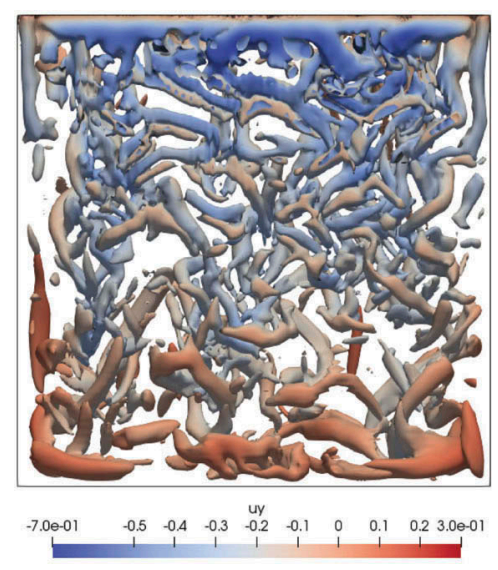

(e) Q-criterion (5) isosurface along front wall, colored by $y$ velocity.

Figure 11. Re 8000 time-averaged $z$-centre plane and instantaneous isosurface results. 


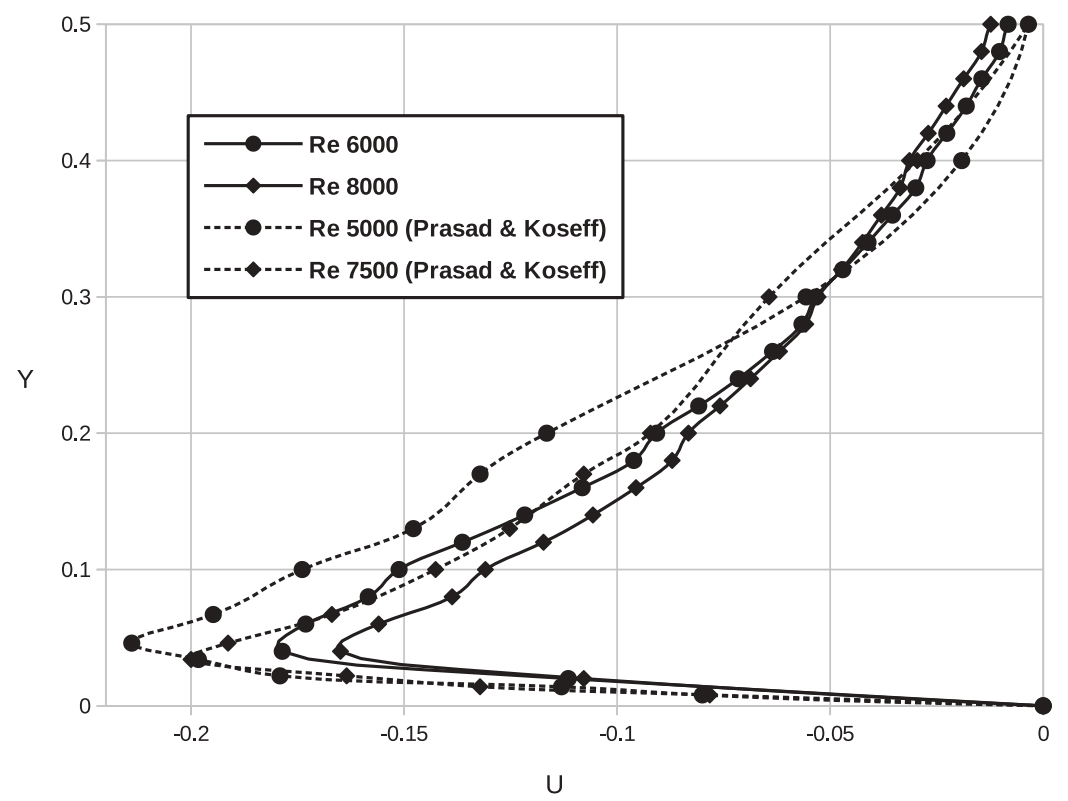

Figure 12. Time-averaged $x$-velocity along lower centreline at transition $R e$ values.

\section{Conclusions}

This work has presented a multiple relaxation time collision operator for a 19 velocity incompressible lattice Boltzmann equation and conducted studies on stability and turbulent transition with a large eddy simulation model. The single relaxation time collision operator is first derived as a basis, and utilises a previously developed ansatz approach. With a generalised constant density of one the resultant multiple relaxation time form deviates from those previously proposed. Low $R e$ lid-driven cavity results verify the derived form behaves in accordance with the incompressible Navier-Stokes equations.

For all cases the Vreman subgrid scale model, an improvement in predicting non-turbulent regimes over the Smagorinsky model, is active. At low-Re the eddy viscosity contribution appropriately scales to allow molecular viscosity to dominate, and on a modest grid allows for an accurate vertical centreline velocity. The onset of laminar instability is also well predicted on a modest grid, with $R e_{\text {Hopf }}=1900-1950$.

By Re 6000 time history, fluctuation magnitude and power spectral density measurements find a turbulent condition near the bottom wall while the core flow is still showing laminar fluctuations. The fluctuation pattern seen in the core has been identified as the meandering of Taylor-Goertler-like vortices through comparison to experimental results. The dual regimes, turbulent near the bottom boundary and unstable laminar in the core corresponds to observation in literature on the progress of transition in the cavity. At Re 8000 turbulent activity arises in the core in the form of intermittent bursts of high-frequency 
random fluctuations. However, the flow still returns to laminar characteristics periodically. The range of $R e 6000-8000$ for transition found here is in general agreement with past literature while adding details to the process. In the fully turbulent zones the characteristics are likewise in agreement with fully turbulent simulations.

Within the framework of an incompressible lattice Boltzmann equation the Vreman subgrid scale model adjusts appropriately in laminar, unstable and transitional regimes.

\section{Notation}

CFD Computational fluid dynamics

$\mathrm{D} d \mathrm{Q} q \quad d$-dimension $q$-velocity lattice architecture

DNS Direct numerical simulation

FVM Finite volume method

LBE $\backslash$ LBE Lattice Boltzmann equation\incompressible form

LBM Lattice Boltzmann method

LDC Lid-driven cavity

LES Large eddy simulation

MRT Multiple relaxation time

NS Navier-Stokes

PSD Power spectral density

$\mathrm{Re} \quad$ Reynolds number

RMS Root mean square

SGS Subgrid scale model

SRS Scale resolving simulation

SRT Single relaxation time

TGL Taylor-Goertler-like

WALE Wall Adapting Local Eddy Viscosity model

\section{Note}

1. The solver was written in the $\mathrm{C}$ language, and parallelised using OpenMP. A MATLAB script for computing equilibrium distribution functions and components required for MRT implementation can be found at https://drive.google.com/file/d/ 1yLfCq7HBcuZglRGe2JWPV7GDJdHfSW64/view?usp=sharing.

\section{Disclosure statement}

No potential conflict of interest was reported by the authors.

\section{ORCID}

J. R. Murdock (D) http://orcid.org/0000-0002-9666-5730

S. L. Yang (D) http://orcid.org/0000-0001-6394-3354 


\section{References}

Anupindi, K., Lai, W., \& Frankel, S. (2014). Characterization of oscillatory instability in lid driven cavity flows using lattice boltzmann method. Computers \& Fluids, 92, 7-21.

Bhatnagar, P., Gross, E., \& Krook, M. (1954). A model for collision processes in gases. I. Small amplitude processes in charged and neutral one-component systems. Physical Review, 94(3), 511-525.

Bouffanais, R., Deville, M., \& Leriche, E. (2007). Large-eddy simulation of the flow in a lid-driven cubical cavity. Physics of Fluids, 19(5), 055108.

Cabot, W. (1994). Local dynamic subgrid-scale models in channel flow (pp. 143-160). Stanford, CA: CTR Annual Research Briefs, Center for Turbulence Research, NASA Ames/Standford University.

Chang, H., Hong, P., Lin, L., \& Lin, C. (2013). Simulations of flow instability in three dimensional deep cavities with multi relaxation time lattice boltzmann method on graphic processing units. Computers \& Fluids, 88, 866-871.

Chen, S., \& Martinez, D. (1996). On boundary conditions in lattice boltzmann methods. Physics of Fluids, 8(9), 2527.

d'Humieres, D. (1992). Generalized lattice boltzmann equations. Progress in Astronautics and Aeronautics, 159, 450-458.

Du, R., \& Shi, B. (2010). Incompressible multi-relaxation-time lattice boltzmann model in 3-d space. Journal of Hydrodynamics, 22(6), 782-787.

Feldman, Y., \& Gelfgat, A. (2010). Oscillatory instability of a 3d lid-driven flow in a cube. Physics of Fluids, 22(9), 093602.

Germano, M., Piomelli, P., Moin, P., \& Cabot, W. (1991). A dynamic subgrid-scale eddy viscosity model. Physics of Fluids A, 3(7), 1760-1765.

Ghia, U., Ghia, K., \& Shin, C. (1982). High-re solutions for incompressible flow using the navier-stokes equations and a multigrid method. Journal of Computational Physics, 48(3), 387-411.

Guo, Z., \& Shu, C. (2013). Lattice boltzmann method and its applications in engineering. Singapore: World Scientific Publishing.

Hammami, F., Ben-Cheikh, N., Campo, A., Ben-Beya, B., \& Lili, T. (2012). Prediction of unsteady states in lid-driven cavities filled with an incompressible viscous fluid. International Journal of Modern Physics C, 23(4), 1250030.

Harris, S. (2011). An introduction to the theory of the boltzmann equation. Mineola, NY: Dover Publications.

Kruger, T., Kusumaatmaja, H., Kuzmin, A., Shardt, O., Silva, G., \& Viggen, E. (2017). The lattice boltzmann method principles and practice. Switzerland: Springer International Publishing.

Kuhlmann, H., \& Albensoeder, S. (2014). Stability of the steady three-dimensional lid-driven flow in a cube and the supercritical flow dynamics. Physics of Fluids, 26(2), 024104.

Ladd, A. (1994). Numerical simulations of particulate suspensions via a discretized boltzmann equation. Part 1. Theoretical foundation. Journal of Fluid Mechanics, 271, 285-309.

Leriche, E., \& Gavrilakis, S. (2000). Direct numerical simulation of the flow in a lid-driven cubical cavity. Physics of Fluids, 12(6), 1363.

Loiseau, J., Robinet, J., \& Leriche, E. (2016). Intermittency and transition to chaos in the cubical lid-driven cavity flow. Fluid Dynamics Research, 48(6), 061421.

Marie, S., Ricot, D., \& Sagaut, P. (2009). Comparison between lattice boltzmann method and navierstokes high order schemes for computational aeroacoustics. Journal of Computational Physics, 228(4), 1056-1070.

MathWorks. (2017). Matlab user's guide. Upper Saddle River, New Jersey: Prentice Hall. 
McNamara, G., \& Zanetti, G. (1988). Use of the boltzmann equation to simulate lattice gas automata. Physical Review Letters, 61(20), 2332-2335.

Ming, L., Xiao-Peng, C., \& Premnath, K. (2012). Comparative study of the large eddy simulations with the lattice boltzmann method using the wall-adapting local eddy-viscosity and vreman subgrid scale models. Chinese Physics Letters, 29(10), 104706.

Murdock, J., Ickes, J., \& Yang, S. (2017). Transition flow with an incompressible lattice boltzmann method. Advances in Applied Mathematics and Mechanics, 9(5), 1271-1288.

Murdock, J., \& Yang, S. (2016). Alternative and explicit derivation of the lattice boltzmann equation for the unsteady incompressible navier-stokes equation. International Journal of Computational Engineering Research, 6(12), 47-59.

Mynam, M., \& Pathak, A. (2013). Lattice boltzmann simulation of steady and oscillatory flows in lid-driven cubic cavity. International Journal for Numerical Methods in Fluids, 24(12), 1340005.

Nicoud, F., \& Ducros, F. (1999). Subgrid-scale stress modelling based on the square of the velocity gradient tensor. Flow, Turbulence and Combustion, 62(3), 183-200.

Pope, S. B. (2000). Turbulent flows. Cambridge, UK: Cambridge University Press.

Prasad, A., \& Koseff, J. (1989). Reynolds number and end-wall effects on a lid-driven cavity flow. Physics of Fluids A, 1(2), 208-218.

Premnath, K., \& Abraham, J. (2007). Three-dimensional multi-relaxation time (mrt) latticeboltzmann models for multiphase flow. Journal of Computational Physics, 224(2), 539-559.

Ren, F., Song, B., \& Haibao, H. (2018). Lattice boltzmann simulations of turbulent channel flow and heat transport by incorporating the vreman model. Applied Thermal Engineering, 129, 463-471.

Shankar, P., \& Deshpande, M. (2000). Fluid mechanics in the driven cavity. Annual Review of Fluid Mechanics, 32, 93-136.

Shen, J. (1991). Hopf bifurcation of the unsteady regularized driven cavity flow. Journal of Computational Physics, 95(1), 228-245.

Shetty, D., Fisher, T., Chunekar, A., \& Frankel, S. (2010). High-order incompressible largeeddy simulation of fully inhomogeneous turbulent flows. Journal of Computational Physics, 229(23), 8802-8822.

Si, H., \& Shi, Y. (2015). Study on lattice boltzmann method/large eddy simulation and its application at high reynolds number flow. Advances in Mechanical Engineering, 7(3), 1-8.

Smagorinsky, J. (1963). General circulation experiments with the primitive equations. Monthly Weather Review, 91, 99-164.

Tolke, J. (2009). Implementation of a lattice boltzmann kernel using the compute unified device architecture developed by nvidia. Computing and Visualization in Science, 13(29), 29-39.

Vreman, A. (2004). An eddy-viscosity subgrid-scale model for turbulent shear flow: Algebraic theory and applications. Physics of Fluids, 16(10), 3670-3681.

Wong, K., \& Baker, A. (2002). A 3d incompressible navierstokes velocityvorticity weak form finite element algorithm. International Journal for Numerical Methods in Fluids, 38, 99-123.

\section{Appendix A: MRT derivation}

Starting from the SRT-iLBE

$$
f(\boldsymbol{x}+\delta \boldsymbol{x}, t+\delta t)-f(\boldsymbol{x}, t)=-\frac{1}{\tau}\left(f(\boldsymbol{x}, t)-f^{e q}(\boldsymbol{x}, t)\right)
$$

and introducing the identity matrix $\boldsymbol{I}=\boldsymbol{M}^{-1} \boldsymbol{M}$, the collision process is modified as 


$$
\begin{aligned}
f(x+\delta x, t+\delta t)-f(x, t) & =-\boldsymbol{M}^{-1} \boldsymbol{M} \frac{1}{\tau}\left(f(x, t)-f^{e q}(x, t)\right) \\
& =-\boldsymbol{M}^{-1} \frac{1}{\tau} I\left(\boldsymbol{M} f(x, t)-\boldsymbol{M} f^{e q}(x, t)\right) \\
& =-\boldsymbol{M}^{-1} \boldsymbol{S}\left(\boldsymbol{m}(x, t)-\boldsymbol{m}^{e q}(x, t)\right),
\end{aligned}
$$

where $S=\frac{1}{\tau_{i}} \boldsymbol{I}$, a diagonal matrix of inverse relaxation times. $\boldsymbol{m}$ and $\boldsymbol{m}^{e q}$ are the moment and equilibrium moment vectors defined as $\boldsymbol{M} \boldsymbol{f}$ and $\boldsymbol{M} \boldsymbol{f}^{e q}$, respectively.

The transformation matrix is constructed of rows of orthnogonal basis vectors which are polynomials of the lattice vectors. Here, $\boldsymbol{M}$ is the commonly utilised matrix in previous non-incompressible derivations (Premnath \& Abraham, 2007)

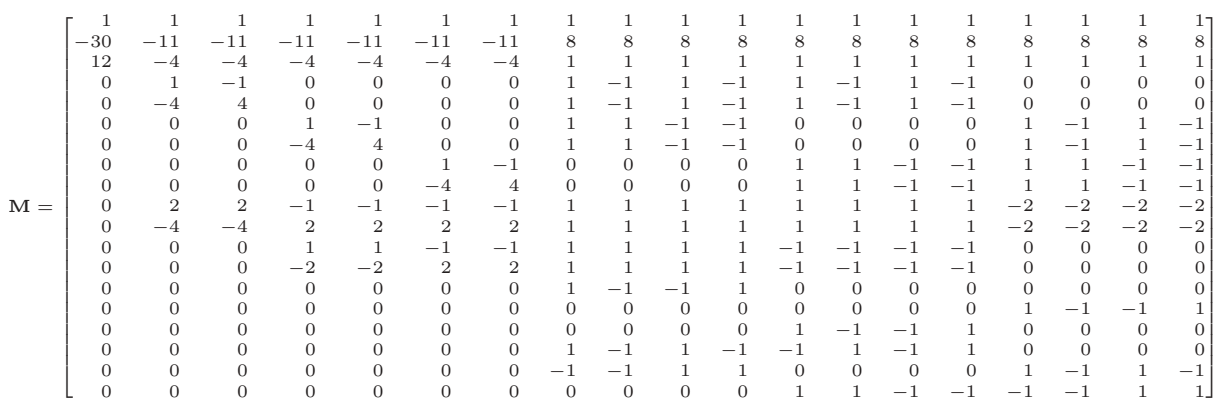

Moment distributions are then defined as

$$
\boldsymbol{m}=\left(\rho_{0}, e, e^{2}, j_{x}, q_{x}, j_{y}, q_{y}, j_{z}, q_{z}, 3 P_{x x}, 3 \pi_{x x}, P_{w w}, \pi_{w w}, P_{x y}, P_{y z}, P_{x z}, m_{x}, m_{y}, m_{z}\right)^{T}
$$

where $m_{0}$ is the density, $m_{1,2}$ are related to energy and energy squared, $m_{3,5,7}$ are components of momentum, $m_{4,6,8}$ are components of energy flux, $m_{9,11,13-15}$ are strain rate tensor-related components, $m_{10,12}$ are fourth-order moments, and $m_{16-18}$ are $3^{\text {rd }}$ order moments.

As in the SRT form, it is the equilibrium distributions which dictate the macroscopic behaviour of the LBE. The array $\boldsymbol{f}^{e q}$ is thus built from Equation (4). Acquiring the array $\boldsymbol{m}^{e q}$ by multiplication of $\boldsymbol{M}$ and $\boldsymbol{f}^{e q}$ is performed with the symbolic computation software MATLAB (MathWorks, 2017) due to the size of the matrices involved. This script can be accessed at https://drive.google.com/file/d/1yLfCq7HBcuZglRGe2JWPV7GDJdHfSW64/ view? usp=sharing. $\boldsymbol{m}^{e q}$ is thus

$$
\begin{array}{ccc}
m_{0}^{e q}=1 & m_{7}^{e q}=u_{z} & m_{14}^{e q}=u_{y} u_{z} \\
m_{1}^{e q}=-30+\frac{19}{3} P+19 u^{2} & m_{8}^{e q}=-\frac{2}{3} u_{z} & m_{15}^{e q}=u_{x} u_{z} \\
m_{2}^{e q}=12-3 P-\frac{11}{2} u^{2} & m_{9}^{e q}=2 u_{x}^{2}-u_{y}^{2}-u_{z}^{2} & m_{16}^{e q}=0 \\
m_{3}^{e q}=u_{x} & m_{10}^{e q}=\frac{1}{2}\left(u_{y}^{2}+u_{z}^{2}\right)-u_{x}^{2} & m_{17}^{e q}=0 \\
m_{4}^{e q}=-\frac{2}{3} u_{x} & m_{11}^{e q}=u_{y}^{2}-u_{z}^{2} & m_{18}^{e q}=0 \\
m_{5}^{e q}=u_{y} & m_{12}^{e q}=\frac{1}{2}\left(u_{z}^{2}-u_{y}^{2}\right) & \\
m_{6}^{e q}=-\frac{2}{3} u_{y} & m_{13}^{e q}=u_{x} u_{y} &
\end{array}
$$


The linked script also computes the transposed transformation matrix and scaling array required for conversion back to velocity space after collisions are performed in moment space.

$S$ allows for unique relaxation times for some elements which can be optimised for stability. To achieve the intended viscosity $s_{9,11,13-15}$ must take on the value $\frac{1}{\tau}$. The other elements can be tuned between 0 and 2 . Unless otherwise stated, the values employed here are

$$
s_{0,3,5,7}=1.0, s_{1}=1.19, s_{2,10,12}=1.0, s_{4,6,8}=1.2, s_{9,11,13,14,15}=\frac{1}{\tau}, s_{16,17,18}=0.98
$$

